\title{
The effects of inclination, gravity darkening and differential rotation on absorption profiles of fast rotators
}

\begin{abstract}
A. Reiners $\star$
Hamburger Sternwarte, Universität Hamburg, Gojenbergsweg 112, 21029 Hamburg, Germany

Received 6 May 2003 / Accepted 20 June 2003

Abstract. Mechanisms influencing absorption line profiles of fast rotating stars can be sorted into two groups; (i) intrinsic variations sensitive to temperature and pressure, and (ii) global effects common to all spectral lines. I present a detailed study on the latter effects focusing on gravity darkening and inclination for various rotational velocities and spectral types. It is shown that the line shapes of rapidly and rigidly rotating stars mainly depend on the equatorial velocity $v_{\mathrm{e}}$, not on the projected rotational velocity $v \sin i$ which determines the lines' widths. The influence of gravity darkening and spectral type on the line profiles is shown. The results demonstrate the possibility of determining the inclination angle $i$ of single fast rotators, and they show that constraints on gravity darkening can be drawn for stellar samples. While significant line profile deformation occurs in stars rotating as fast as $v_{\mathrm{e}} \gtrsim 200 \mathrm{~km} \mathrm{~s}^{-1}$, for slower rotators profile distortions are marginal. In these cases spectral signatures induced by, e.g., differential rotation are not affected by gravity darkening and the methods applicable to slow rotators can be applied to these faster rotators, too.
\end{abstract}

Key words. line: profiles - stars: rotation

\section{Introduction}

The information contained in line profiles of rotating stars has been studied since the recognition of spectral Doppler broadening itself. The multitude of different mechanisms influencing line profiles presents a confusing picture for the possibility of determining them. Approximations exist for rotational line broadening, (linear) limb darkening, differential rotation, turbulence, rotational flattening and gravity darkening, etc. While distinguishing these interacting mechanisms is a delicate issue, in the case of slow rotators $\left(v \sin i<50 \mathrm{~km} \mathrm{~s}^{-1}\right)$ rotational flattening and gravity darkening can be neglected. Utilizing the Fourier transform technique Gray $(1973,1976)$ also showed that turbulent velocities to some extent can be distinguished from rotation.

In fast rotators the situation becomes more complex, since centrifugal forces distort the spherical shape of the stellar surface and the line profiles depend on the inclination the star is observed under. Beyond the additional degree of freedom this would not be a major problem if temperature (and pressure) were not connected to gravity. With different regions on the stellar surface having significantly different temperatures and gas pressures, line profiles no longer can be approximated by convolution between a single intrinsic line profile and the rotational broadening function. For this reason, the effects of fast rotation on line profiles often were studied for specific absorption lines (e.g., Stoeckley 1968; Hardorp \& Strittmatter 1968);

\footnotetext{
* e-mail: areiners@hs.uni-hamburg.de
}

Collins \& Truax (1995) come to the conclusion that the detection of secondary effects like limb darkening and differential rotation is improbable in line profiles of fast rotators.

Under what circumstances the approximation of various broadening mechanisms by convolutions breaks down, only depends on the sensitivity of the spectral line on temperature and gas pressure. Spectral quality in terms of signal-to-noise as well as in wavelength coverage has improved and makes possible analyses of weak absorption features. Observers are not restricted to the extremely temperature and pressure sensitive lines of the lightest elements $\mathrm{H}$ and $\mathrm{He}$. Fast rotation is the rule in stars of spectral types as late as F5, and in the spectra of fast rotating A- and F-stars a large number of heavy ion lines exist where the approximation of rotational broadening by a convolution becomes valid again. Especially the deconvolution of an "overall" broadening function inherent in all lines by using the methods of Least Squares Deconvolution (LSD, e.g., Cameron 2000) can provide reliable broadening profiles independent of line specific intrinsic mechanisms.

In this paper the effects of fast rotation are examined in detail focusing on geometric distortions and gravity darkening on stars observed under different inclination angles. These effects underly all spectral lines independent of their intrinsic shape and temperature or pressure dependencies. The modelled spectra assume no intrinsic temperature or pressure dependence; whether this can be applied to specific spectral lines or groups of lines has to be checked individually. 
In Reiners \& Schmitt (2002a) the possibility of detecting differential rotation in line profiles was demonstrated. This paper also answers the question of whether the technique used there is applicable to fast rotators, too. It shows what can be learned about gravity darkening and inclination angle from the shape of stellar absorption line profiles.

\section{Absorption profiles of fast rotators}

\subsection{Line-independent broadening}

Absorption line profiles observed in fast rotating stars are affected by three mechanisms inherent in all lines: (a) the projected geometry of the star, (b) the flux distribution due to temperature variations (known as gravity darkening) on the rotating stellar surface, and (c) the rotation law. These three lineindependent mechanisms will be discussed in the following.

Case (a) is well understood, fast rotation diminishes the gravitational potential at the equator and alters the star's sphericity. As a consequence, lines of constant projected rotational velocities no longer lie on chords but are bent (cp., e.g., Collins \& Truax 1995). Although an analytical description of the surface velocity distribution is not then available, calculation by integrating over the stellar surface is unproblematic.

(b) Since the presence of gravity darkening was initially described by von Zeipel (1924), many publications were written on the correct gravity dependence of surface temperature. In general, gravity darkening is described in terms of a parameter $\beta$ used in the equation

$T_{\text {eff }} \propto g^{\beta}$.

The "classical" value of $\beta=0.25$ found by von Zeipel (1924) is valid only for conservative rotation laws in stars without convective envelopes. A value of $\beta=0.08$ is derived for stars with convective envelopes (Lucy 1967). Tuominen (1972) approximates a value of $\beta \approx 0.15$ for differentially rotating stars, and for magnetic stars without convection zones, Smith (1975) reports a slightly different value of $\beta=0.275$. The most comprehensive calculations were carried out by Claret (1998), who provides tables of stellar parameters including $\beta$ for a series of stars at various evolutionary stages. The calculations are in general agreement with the cited works; stars of spectral types later than $\sim \mathrm{A} 2$ harboring convective envelopes have a value of $\beta \approx 0.08$, for earlier-type stars, $\beta=0.25$ seems a good choice. These calculations are also consistent with measurements of $\beta$ in eclipsing binaries carried out by Rafert \& Twigg (1980), although a large scatter appears in the data (cp. Fig. 7 in Claret 1998).

(c) Stellar rotation laws are poorly known. Our picture comes from observations of the Sun, where the angular velocity can be approximated as

$\Omega(l)=\Omega_{\text {Equator }}\left(1-\alpha \sin ^{2} l\right)$,

with $l$ being the latitude and $\alpha_{\odot} \approx 0.2$, i.e., the Equator rotating $20 \%$ faster than the Pole as derived from Sun spots. Differential rotation in stars other than the Sun was claimed for ten relatively slowly rotating F-type stars with values of $v \sin i<$ $50 \mathrm{~km} \mathrm{~s}^{-1}$ in Reiners \& Schmitt (2003) adopting Eq. (2) as the rotation law in analogy to the solar case. Assuming reasonable values of inclination angles $i$, the small projected rotational velocities of the stars in that sample give no reason to expect effects due to distortions of the gravitational potential, and differential rotation is thought to be the cause of the determined profile signatures. It is an open question, to what extent differential rotation can be detected in faster rotators, where deformation and gravity darkening become important.

\subsection{Profile shape characteristics}

Stellar line profiles are subject to a number of different mechanisms affecting their shape. Besides the mentioned effects of gravity darkening and rotation law, limb darkening, additional (turbulent) velocity fields and stellar surface structure like spots can significantly contribute to the line profiles' shape. Utilizing the Fourier transform turns out to be of great advantage in disentangling the different effects. When approximating line broadening by convolutions, their interaction can be studied most easily using Fourier transforms, since the computationally complicated convolutions become multiplications in Fourier domain (cf., e.g., Gray 1976).

Reiners \& Schmitt (2002a) utilized the zeros of the Fourier transformed line profiles to search for the subtle effects of differential rotation. They showed that the ratio of the first two zeros $q_{1}$ and $q_{2}$ is a direct indicator for solar-like - i.e., Equator faster than Pole - differential rotation ( $\alpha>0$ in Eq. (2)). The value of the ratio $q_{2} / q_{1}$ is extremely sensitive to the profile's shape, and is unaffected by the "standard" turbulent velocity fields like micro- and macroturbulence even for rotational velocities as low as $10 \mathrm{~km} \mathrm{~s}^{-1}$. Using a linear limb darkening law with a parameter $\epsilon$, in a rigidly rotating star the value of $q_{2} / q_{1}$ can be approximated as

$$
\frac{q_{2}}{q_{1}}=1.831-0.108 \epsilon-0.022 \epsilon^{2}+0.009 \epsilon^{3}+0.009 \epsilon^{4}
$$

(Dravins et al. 1990). Thus for a rigid rotator the value of $q_{2} / q_{1}$ is always between 1.72 and 1.83 regardless of the limb darkening coefficient. Differential rotation significantly diminishes this ratio (cf Reiners \& Schmitt 2002a), while polar spots lead to an increase (Reiners \& Schmitt 2002b).

Since the ratio $q_{2} / q_{1}$ turns out to be an easily accessible parameter characteristic for the line's shape, I continue to use it for studying the influence of gravity darkening in rapidly rotating stars. For a more detailed discussion of the correlations between differential rotation $\alpha$, the inclination angle $i$ and $q_{2} / q_{1}$ see Reiners \& Schmitt (2002a, 2003).

\section{Model}

The approach of this paper is to study the effects of fast rotation universal for all absorption lines. Line specific dependencies on gas pressure, ionization stages, etc. are not taken into account. This approach is especially suited for studies incorporating different lines of similar (preferably heavy) ions, e.g., with LSD methods. For heavy ions pressure broadening is not expected to play a major role compared to the dominant rotational broadening. For light elements like $\mathrm{H}$ and $\mathrm{He}$, this is not 
Table 1. Parameters of the calculated models, $a$ and $b$ are parameters used in Eq. (4) with values of gravity darkening $\beta$ according to Claret (1998). Note that for all models profiles with $\beta=0.0,0.08$ and 0.25 were analyzed.

\begin{tabular}{crrccc}
\hline \hline Model & $T_{\text {eff }}^{\text {Pole }}$ & $M / M_{\odot}$ & $\beta$ & $a$ & $b$ \\
\hline B0 & 30000 & 17.5 & 0.25 & $.740 \mathrm{E}-4$ & $-.345 \mathrm{E}-6$ \\
A0 & 10000 & 2.4 & 0.25 & $.190 \mathrm{E}-3$ & $-.136 \mathrm{E}-5$ \\
F0 & 7000 & 1.6 & 0.08 & $.172 \mathrm{E}-3$ & $-.993 \mathrm{E}-6$ \\
G0 & 5700 & 1.0 & 0.08 & $.184 \mathrm{E}-3$ & $-.116 \mathrm{E}-5$ \\
\hline
\end{tabular}

true and great care has to be take when applying the methods to them. Although the Lorentzian profiles induced by pressure broadening should not add additional zeros to the line profile Fourier transforms (cp. Heinzel 1978), errors from approximating line broadening effects by convolutions may become significant in these cases.

The calculations were done using a modified version of the package developed and described by Townsend (1997). Surface integration is carried out over 25500 visible surface elements scaling the flux with temperature according to a Planck law. A Gaussian profile was used as the input function, since the large rotational velocities make the shape of the input function unimportant. For all cases the same input function was used regardless of gravity and temperature. To measure the zeros in Fourier transformed line profiles the accuracy must be higher for the faster rotators; 32768 points were used in the transform algorithm for values of $v \sin i \leq 100 \mathrm{~km} \mathrm{~s}^{-1}, 65536$ points for values of $v \sin i$ between 100 and $200 \mathrm{~km} \mathrm{~s}^{-1}$, and 131072 points for $v \sin i>200 \mathrm{~km} \mathrm{~s}^{-1}$. Thus the sampling error in $q_{2} / q_{1}$ does not exceed a value of 0.01 for all calculations.

Broadening profiles are calculated and the values of $q_{2} / q_{1}$ determined for four stellar models with polar effective temperature $T_{\text {eff }}^{\text {Pole }}$ and mass given in columns two and three in Table 1. A grid is computed in three values of $\beta(0.0,0.08$ and 0.25$)$, five values of inclination angle $i\left(10^{\circ}, 30^{\circ}, 50^{\circ}, 70^{\circ}\right.$ and $90^{\circ}$ ), and values of $v \sin i$ between 50 and $300 \mathrm{~km} \mathrm{~s}^{-1}$ in steps of $25 \mathrm{~km} \mathrm{~s}^{-1}$ with the equatorial velocity $v_{\mathrm{e}}<350 \mathrm{~km} \mathrm{~s}^{-1}$. Altogether more than 450 models are computed. The dependence of the ratio $q_{2} / q_{1}$ on different parameter choices is analyzed and studied in the following.

\section{Line shape variations}

\subsection{Monotonic dependence on rotational velocity $v_{e}$}

To show the dependence of $q_{2} / q_{1}$ on $v_{\mathrm{e}}$, in Fig. $1 q_{2} / q_{1}$ is plotted vs. $v_{\mathrm{e}}$ for the $\mathrm{F} 0$ model with a gravity darkening parameter of $\beta=0.08$. The region in $q_{2} / q_{1}$ typical for a spherical surface and a linear limb darkening law with $0.0<\epsilon<1.0$ is indicated with dotted lines (cp. Eq. (3)). Values for stars seen under various inclination angles are distinguished by different symbols, all values are in good agreement with the marked polynomial indicating a smooth decline of $q_{2} / q_{1}$ for stars rotating faster than $v_{\mathrm{e}} \approx 150 \mathrm{~km} \mathrm{~s}^{-1}$.

The parameter determining the value of $q_{2} / q_{1}$ in a given stellar model obviously is $v_{\mathrm{e}}$, independent of the inclination

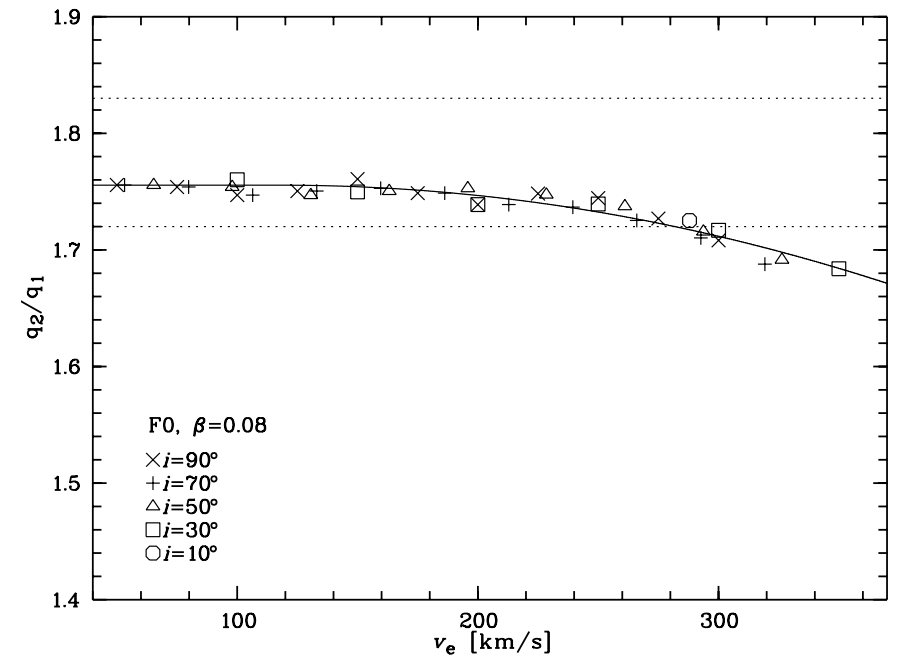

Fig. 1. Derived values of $q_{2} / q_{1}$ for a rigidly rotating F0-type star with $\beta=0.08$. Different symbols indicate inclination angles as indicated in the figure. The value of $q_{2} / q_{1}$ is determined by $v_{\mathrm{e}}$ independent of the values of $i$ and $v \sin i$. The slope is well approximated by the second order polynomial shown as a solid line.

angle (and thus $v \sin i$ ). This means, that under the assumption of rigid rotation, and with assumed values of $\beta$, in fast rotators one can in principle obtain the value of $v_{\mathrm{e}}$ from the value of $q_{2} / q_{1}$, and thus, with the known value of $v \sin i$, the inclination angle $i$. I will return to this point in Sect. 4.3.

\subsection{Gravity darkening and spectral class}

In the four panels of Fig. 2 the derived values of $q_{2} / q_{1}$ are plotted vs. $v_{\mathrm{e}}$ for the models of spectral types B0, A0, F0 and G0 as specified in Table 1. In each panel calculations for three different values of $\beta$ are shown, the different choices of $\beta$ (0.0, 0.08 and 0.25 ) are plotted using different symbols. The slope of $q_{2} / q_{1}$ is well described by a second order polynomial for all cases. The ratio $q_{2} / q_{1}$ is diminished in rapid rotators with larger deviations from the standard value of $q_{2} / q_{1}=1.75$ the larger the value of $\beta$. In the A0, F0 and G0 models, $q_{2} / q_{1}$ becomes less than 1.72 for very fast rotators depending on the value of $\beta$, i.e., deviations from standard line profiles are significant and in principle observable in these stars.

The behavior of $q_{2} / q_{1}$ with different values of the gravity darkening parameter $\beta$ can be studied in the four panels of Fig. 2. In the B0 model no value of $q_{2} / q_{1}<1.72$ appears, but the influence of $\beta$ on the ratio $q_{2} / q_{1}$ grows with later spectral type. In all cases $q_{2} / q_{1}$ depends monotonically on the rotational velocity $v_{\mathrm{e}}$ with diminished $q_{2} / q_{1}$ for larger rotational velocities. The result that the line profiles depend more strongly on the value of $v_{\mathrm{e}}$ for stars of later spectral types, reflects the fact that the relative temperature contrast $\Delta T / T$ on the stellar surface is larger for cooler stars, since $\Delta T$ is comparable for similar values of $v_{\mathrm{e}}$. Furthermore, for all models no significant variation of $q_{2} / q_{1}$ appears for $\beta=0$, i.e., without gravity darkening the geometrical deformation does not suffice to significantly change the shape of the line profiles. 

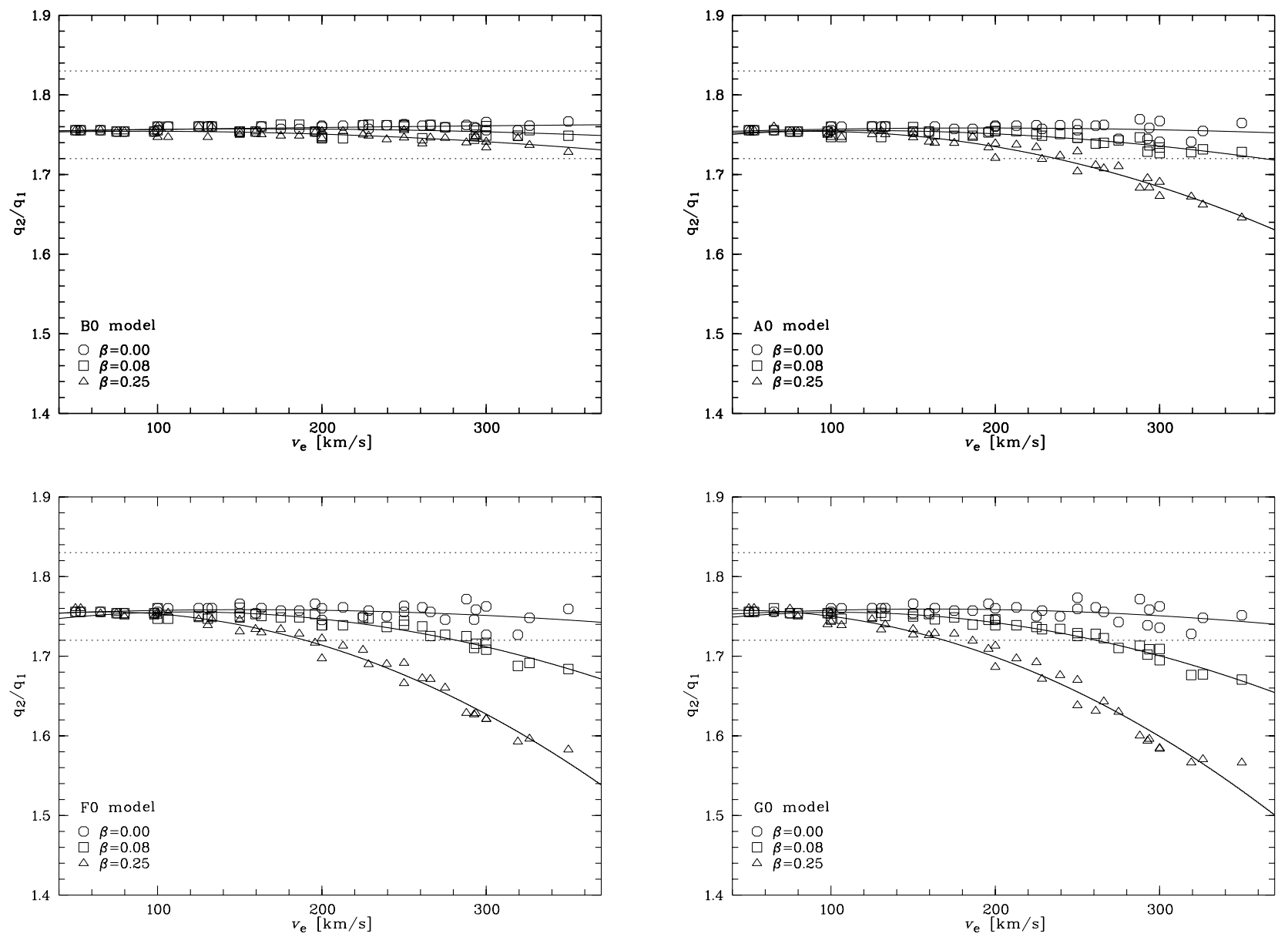

Fig. 2. Derived values of $q_{2} / q_{1}$ vs. equatorial velocity $v_{\mathrm{e}}$ for four different stellar models (B0, A0, F0 and G0). For each model different values of the gravity darkening parameter $\beta(0.0,0.08$ and 0.25$)$ are indicated using different symbols. The dependence of $q_{2} / q_{1}$ on $v_{\mathrm{e}}$ differs for the different cases but is monotonic in $\beta$ and $v_{\mathrm{e}}$.

In a larger sample of stars of similar spectral types these results could make it feasible to determine the value of gravity darkening in terms of the parameter $\beta$ when plotting $q_{2} / q_{1}$ vs. $v \sin i$. While for many stars in such a plot the values should fall below an upper envelope expected from the $q_{2} / q_{1}-v_{\mathrm{e}}$ relation (the real values of $v_{\mathrm{e}}$ are larger than the measured values of $v \sin i$ ), a clear threshold should mark the upper envelope indicating the correct value of $\beta$. Using a larger sample of fast rotators of similar spectral types the ratio $q_{2} / q_{1}$ could thus be used to determine the values of $\beta$ from measurements of single stars.

\subsection{Determination of $i$}

The ratio $q_{2} / q_{1}$ monotonically depends on equatorial velocity $v_{\mathrm{e}}$ and is independent of the inclination angle $i$. Assuming a value of $\beta$ for a fast rotator of known spectral class, the equatorial velocity $v_{\mathrm{e}}$ can thus be determined from a measurement of $q_{2} / q_{1}$. The functions $q_{2} / q_{1}\left(v_{\mathrm{e}}\right)$ shown in Fig. 2 are approximated by the second order polynomial

$q_{2} / q_{1}=1.75+a v_{\mathrm{e}}+b v_{\mathrm{e}}^{2}$.

The parameters $a$ and $b$ for the four models using the most probable values of the parameter $\beta$ according to Claret (1998) are given in Table 1. With Eq. (4) it is possible to derive the inclination angle $i$ from a standard measurement of $v \sin i$ and the value of $q_{2} / q_{1}$.

In each panel of Fig. 3 the values of $i$ are plotted vs. the ratio $q_{2} / q_{1}$ for four different values of $v \sin i$. As in Fig. 2 each panel represents a stellar model from Table 1. Functions for values of $v \sin i=50,100,200$ and $300 \mathrm{~km} \mathrm{~s}^{-1}$ are plotted as examples; for calculating intermediate values of $v \sin i$, Eq. (4) can be used with values of $a$ and $b$ as given in columns five and six of Table 1. For example, a value of $q_{2} / q_{1}=1.70$ measured in a profile of an A0-type star with $v \sin i=100 \mathrm{~km} \mathrm{~s}^{-1}$ can indicate that a rigidly rotating star is observed under an inclination of $i=20^{\circ}\left(v_{\mathrm{e}} \approx 290 \mathrm{~km} \mathrm{~s}^{-1}\right)$.

\subsection{Differential rotation in fast rotators}

Solar-like differential rotation with the Equator faster than the Pole diminishes the value of $q_{2} / q_{1}$ as shown in Reiners \& Schmitt (2002a); a measurement yielding $q_{2} / q_{1}<1.72$ can be due to a solar-like rotation law with the Equator faster than the Pole. Whether very fast rotators are subject to strong differential rotation will not be discussed here. In Fig. $2 q_{2} / q_{1}$ appears to be larger than 1.72 for all stars with $v_{\mathrm{e}}<200 \mathrm{~km} \mathrm{~s}^{-1}$ and values of gravity darkening according to Claret (1998) (cp. 

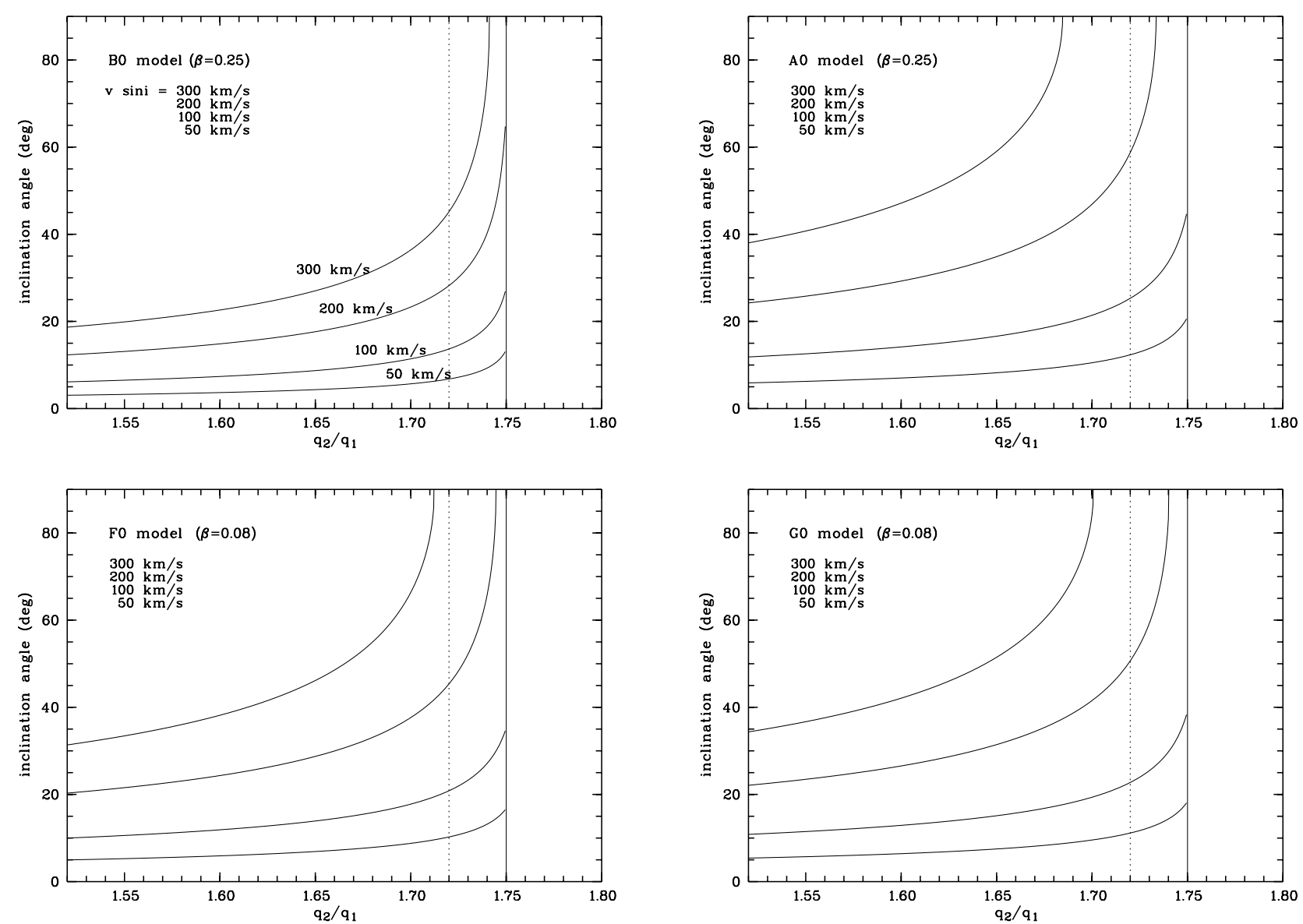

Fig. 3. Stars with measured values of $v \sin i$ and $q_{2} / q_{1}$ are seen under an inclination angle $i$. In the four panels (B0, A0, F0 and G0-type stars) the approximated dependence of $i$ on the ratio $q_{2} / q_{1}$ is shown for values of $v \sin i=50,100,200$ and $300 \mathrm{~km} \mathrm{~s}^{-1}$ (solid lines, top to bottom as indicated in the upper left panel). The dashed line marks the lowest ratio of $q_{2} / q_{1}$ consistent with slow rotation and extreme limb darkening, no values of $q_{2} / q_{1}>1.75$ are expected.

Table 1). This means that a measurement of differential rotation by determining the ratio $q_{2} / q_{1}$ is not affected by gravity darkening if $v_{\mathrm{e}} \lesssim 200 \mathrm{~km} \mathrm{~s}^{-1}$. Interpreting gravity darkening as the reason for a measured value of $q_{2} / q_{1}<1.72$, the rotational velocity $\left(v_{\mathrm{e}}>200 \mathrm{~km} \mathrm{~s}^{-1}\right)$ and inclination angle of the star can be determined. Consistency of $v_{\mathrm{e}}$ with breakup velocity can be checked. For $v_{\mathrm{e}}<v_{\text {crit }}$, differential rotation and very fast rotation remain indistinguishable without further information. An example is given in Sect. 6.

\section{Real and measured values of $v \sin i$}

To what accuracy a measurement of $v \sin i$ is possible was discussed in a number of publications (e.g., Stoeckley 1968; Collins \& Truax 1995). It is known that measured values of $v \sin i$ underestimate the real values especially for very fast rotators regardless of the data quality. To what extent a measured value is too low, depends on the gravity darkening law and on the stellar inclination. In the four panels of Fig. 4 the differences between real and measured values for the B0, A0, F0 and G0-type stars are shown using the most probable values of gravity darkening as done above. The measured values of $v \sin i$ have been derived using the first zeros of the Fourier transform. Values for different inclination angles are differentiated and shown with different symbols, those derived for identical values of $i$ are connected by solid lines. Dashed lines mark the identity of real and measured values. As expected, the measured values of $v \sin i$ may underestimate the real ones but will never indicate too high a projected rotational velocity. This reflects the fact that the (equatorial) surface regions with the largest projected rotational velocities become cooler due to gravity darkening, and thus less flux is observed from them.

The extent, to which a measured value of $v \sin i$ is systematically underestimated, depends strongly and in a welldefined manner on the inclination angle. For constant inclination angles, the measured value of $v \sin i$ is a smooth function of the real one (cp. to Fig. 6 in Collins \& Truax 1995). Underestimation of $v \sin i$ is stronger for smaller inclination angles in all models. The deviation naturally is higher for large values of $v \sin i$ (and thus also of $v_{\mathrm{e}}$ ). Thus, measured values of $v \sin i$ in very rapid rotators can be corrected by determining the ratio $q_{2} / q_{1}$ and calculating the inclination angle $i$ as shown in Sect. 4.3.

It should be noted that - in contrast to Fig. 12 in Collins \& Truax (1995) - inclination has a strong effect on the zeros of Fourier transformed line profiles of rapid rotators; the case shown there only holds for zero projected rotational velocity. 

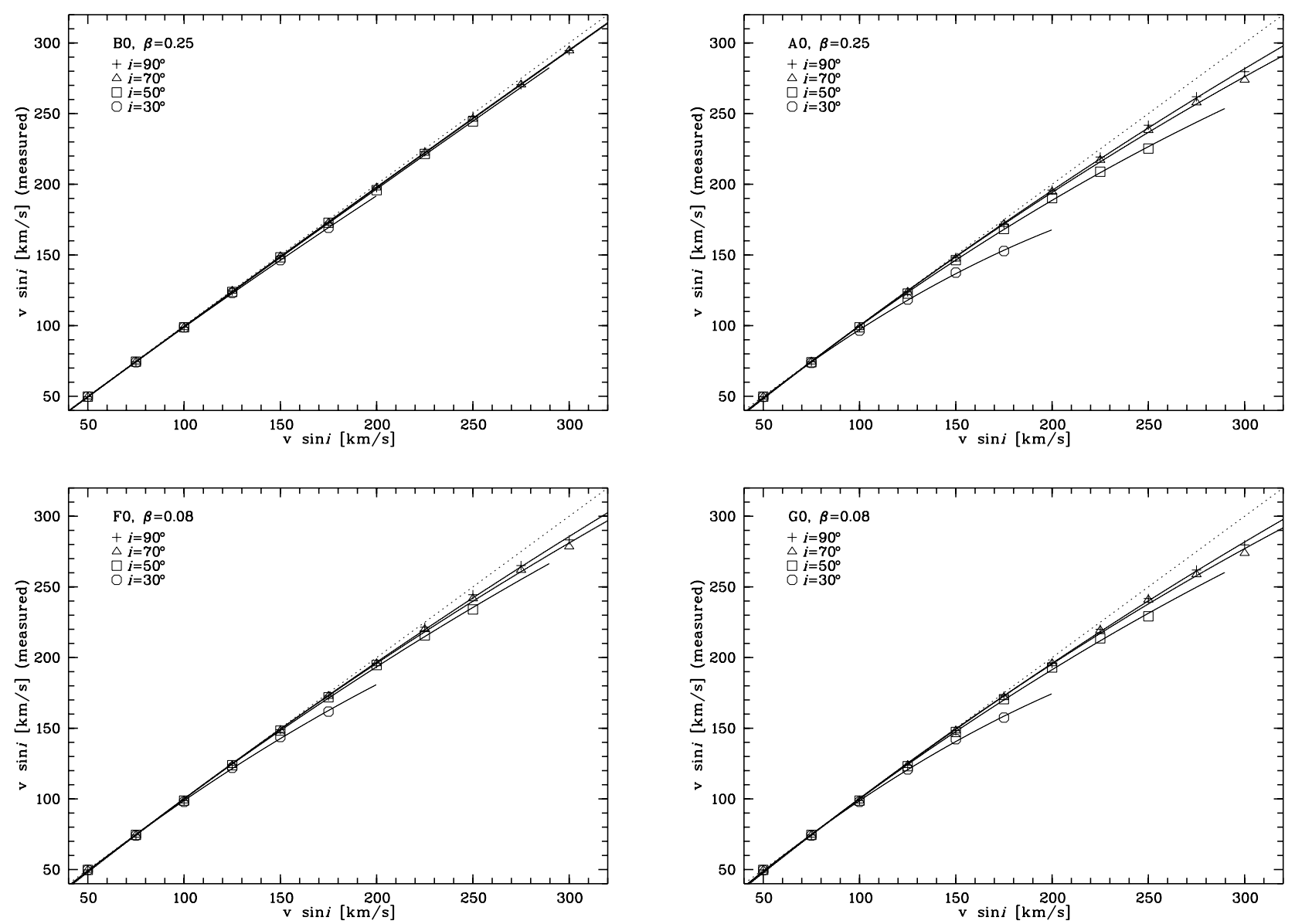

Fig. 4. Values of $v \sin i$ as measured from the first zero of the Fourier transformed broadening profiles plotted vs. the real values given as input to the models. The four panels show cases of $\mathrm{B} 0, \mathrm{~A} 0, \mathrm{~F} 0$ and $\mathrm{G} 0$-type stars, the value of the gravity darkening parameters $\beta$ are chosen according to Claret (1998). In each panel series for four different inclination angles $\left(i=90^{\circ}, 70^{\circ}, 50^{\circ}\right.$ and $\left.30^{\circ}\right)$ are differentiated, the respective values are connected by solid lines. Dotted lines mark the identity between real and measured values.

\section{Example: Differential or fast rotation?}

As an observational example the ten stars for which differential rotation was claimed in Reiners \& Schmitt (2003) are given in Table 2 with their determined values of $v \sin i$ and $q_{2} / q_{1}$. According to the present calculations, these values of $q_{2} / q_{1}<$ 1.72 could also be interpreted as fast rotation seen under small inclination angles $i$. The required values of $v_{\mathrm{e}}$ and $i$ for this interpretation are calculated and given in columns five and six in Table 2. With one exception all values of $v_{\mathrm{e}}$ are larger than break-up velocity and thus can be excluded (it is furthermore very unlikely to find inclination angles below $6^{\circ}$ in ten out of the 32 objects used in that sample). Thus differential rotation remains the most probable explanation for the peculiar shapes of these profiles.

\section{Summary}

Line independent effects of fast stellar rotation on line profiles were presented. Neglecting line specific variations in shape and line depth, the effects of geometrical deformation and the role of the gravity darkening parameter $\beta$ have been outlined. Grid calculations in gravity darkening $\beta$, inclination angle $i$, rotational velocity $v_{\mathrm{e}}$ and spectral type with the parameter $q_{2} / q_{1}$
Table 2. Stars with measured values $q_{2} / q_{1}<1.72$ from the sample of Reiners \& Schmitt (2003); differential rotation is claimed for these objects. Interpreting the values of $q_{2} / q_{1}$ as due to very fast rotation seen under small inclination angles, the required values of $v_{\mathrm{e}, \text {,rigid }}$ and $i$ are given. Nine of the ten are larger than break-up velocity.

\begin{tabular}{rlcccc}
\hline \hline HD & Type & $\begin{array}{c}v \sin i \\
{\left[\mathrm{~km} \mathrm{~s}^{-1}\right]}\end{array}$ & $q_{2} / q_{1}$ & $\begin{array}{c}v_{\text {e,rigid }} \\
{\left[\mathrm{km} \mathrm{s}^{-1}\right]}\end{array}$ & $i$ \\
\hline 89449 & F6IV & $17.3 \pm 1.7$ & 1.44 & 630 & $1.7^{\circ}$ \\
89569 & F6V & $12.2 \pm 0.7$ & 1.57 & 500 & $1.4^{\circ}$ \\
100563 & F5V & $13.5 \pm 0.4$ & 1.67 & 370 & $2.1^{\circ}$ \\
105452 & F0IV/V & $23.5 \pm 1.2$ & 1.59 & 500 & $2.7^{\circ}$ \\
120136 & F7V & $15.6 \pm 1.0$ & 1.57 & 500 & $1.8^{\circ}$ \\
121370 & G0IV & $13.5 \pm 1.3$ & 1.46 & 590 & $1.3^{\circ}$ \\
160915 & F6V & $12.4 \pm 0.5$ & 1.60 & 470 & $1.5^{\circ}$ \\
173667 & F6V & $18.0 \pm 2.0$ & 1.40 & 660 & $1.6^{\circ}$ \\
175317 & F5IV/V & $17.1 \pm 0.7$ & 1.58 & 490 & $2.0^{\circ}$ \\
197692 & F5V & $41.7 \pm 1.7$ & 1.62 & 440 & $5.4^{\circ}$ \\
\hline
\end{tabular}

classifying the profiles' shapes were carried out. The results summarized below can be applied especially to high quality data covering large wavelength regions where line-independent broadening profiles may be obtained using the methods of 
LSD. Applicability must be proved for each case individually, but especially for lines of heavier ions in stars of later spectral types, intrinsic variations of line profiles are sufficiently small and the results of this study can directly be applied. The results can be summarized as follows:

1. In very fast rotators $\left(v \sin i>150 \mathrm{~km} \mathrm{~s}^{-1}\right)$ and for constant values of $\beta$ and similar spectral types, the value of $q_{2} / q_{1}$ shows monotonic dependence on equatorial velocity $v_{\mathrm{e}}$ with $q_{2} / q_{1}$ diminishing significantly with larger values of $v_{\mathrm{e}}$. Variations with inclination $i$ are marginal.

2. The coupling of $q_{2} / q_{1}$ on $v_{\mathrm{e}}$ depends on the value of the gravity darkening parameter $\beta$ and of spectral type. Larger values of $\beta$ lead to lower values of $q_{2} / q_{1}$ with stronger slopes for later spectral types. Estimating the physical values of $\beta$ according to Claret (1998), the parameter $q_{2} / q_{1}$ becomes significantly diminished in fast rotating $\left(v_{\mathrm{e}} \gtrsim\right.$ $200 \mathrm{~km} \mathrm{~s}^{-1}$ ) A-, F- and G-type stars. Independent of the inclination angle $i$, the equatorial velocity $v_{\mathrm{e}}$ can be estimated by measuring $q_{2} / q_{1}$, and thus measuring $v \sin i$ and $v_{\mathrm{e}}\left(q_{2} / q_{1}\right)$ determines the inclination angle $i$.

3. For stars with rotational velocities of $v_{\mathrm{e}} \lesssim 200 \mathrm{~km} \mathrm{~s}^{-1}$ the value of the ratio $q_{2} / q_{1}$ does not significantly change and is larger than 1.72 for all choices of $\beta$ and for all spectral types.

4. For fixed values of $v \sin i$ the zeros of Fourier transformed rotational broadening profiles do depend on inclination angle $i$. A smaller value of $i$ results in a larger difference between the value of $v \sin i$ as measured from the first zero of the Fourier transform and its real value. Measured projected rotational velocities are never overestimated. Deviations are as large as $20 \%$ depending on the value of $i$. Using the parameter $q_{2} / q_{1}$ this effect can be corrected for.
As for differential rotation, it turned out that its measurement is not affected by gravity darkening in stars rotating slower than $v_{\mathrm{e}} \approx 200 \mathrm{~km} \mathrm{~s}^{-1}$. For faster rotators, small values of $q_{2} / q_{1}$ can principally be due to three reasons; (i) strong differential rotation, (ii) very fast rigid rotation (perhaps seen under a small inclination angle), and (iii) both. The constraints on a value of $v_{\mathrm{e}}\left(q_{2} / q_{1}\right)$ can help to clarify the situation, e.g., if a value of $v_{\mathrm{e}}$ larger than breakup velocity is needed to rule out differential rotation.

Acknowledgements. A.R. acknowledges financial support from Deutsche Forschungsgemeinschaft DFG-SCHM 1032/10-1.

\section{References}

Cameron, A. C. 2000, Lect. Note Phys., 573, 183

Claret, A. 1998, A\&AS, 131, 395

Collins, G. W. II, \& Truax, R. J. 1995, ApJ, 439, 860

Dravins, D., Lindegren, L., \& Torkelsson, U. 1990, A\&A, 237, 137

Gray, D. F. 1973, ApJ, 184, 461

Gray, D. F. 1976, The observation and analysis of stellar photospheres (New York: Wiley)

Hardorp, J., \& Strittmatter, P. A. 1968, ApJ, 153, 465

Heinzel, P. 1978, Bull. Astron. Inst. Czechosl., 29, 159

Lucy, L. B. 1967, Z. Astrophys., 65, 89

Rafert, J. B., \& Twigg, L. W. 1980, MNRAS, 193, 79

Reiners, A., \& Schmitt, J. H. M. M. 2002a, A\&A, 384, 555

Reiners, A., \& Schmitt, J. H. M. M. 2002b, A\&A, 388, 1120

Reiners, A., \& Schmitt, J. H. M. M. 2003, A\&A, 398, 647

Smith, R. C. 1975, MNRAS, 173, 97

Stoeckley, T. R. 1968, MNRAS, 140, 121

Townsend, R. 1997, MNRAS, 284, 839

Tuominen, I. V. 1975, Ann. Acad. Sci. Fennicae, Ser. A, VI. Physica, 393 (3.)

von Zeipel, H. 1924, MNRAS, 84, 655 